\title{
Investigating the clinical significance of body composition changes in patients undergoing chemoradiation for oropharyngeal cancer using analytic morphomics
}

\author{
Chen Wang ${ }^{1}$, Jeffrey M. Vainshtein ${ }^{1}$, Maria Veksler², Patrick E. Rabban², June A. Sullivan², Stewart C. Wang ${ }^{2}$, \\ Avraham Eisbruch ${ }^{1}$ and Shruti Jolly ${ }^{1,3^{*}}$
}

\begin{abstract}
Background: The purpose is to investigate the clinical significance of body morphomics changes in stage III-IV oropharyngeal cancer patients during concurrent chemoradiotherapy (CRT).

Methods: Fifty patients who underwent CRT were selected for body composition analyses by either availability of pre/post treatment DEXA scans or a novel CT-based approach of body morphomics analysis (BMA). BMA changes (lean psoas and total psoas area) were compared to total lean body mass changes by DEXA scans using two-sample $t$ tests. Pearson correlation was used to compare the BMA measures to head and neck specific quality of life outcomes. Cox hazards model was used to predict mortality and tumor recurrence.

Results: Clinically significant declines in total psoas area and lean body mass of similar magnitude were observed in both BMA and DEXA cohorts after CRT. Loss of psoas area $(P<0.05)$ was associated with greater frailty and mobility issues ( 3 out of 15 UWQOL domains). Total psoas area is more sensitive for local recurrence than weight changes and T-stage on multivariate analyses.
\end{abstract}

Conclusions: BMA specifically evaluating psoas area appears to correlate with head and neck cancer quality of life physical domains. Pre- and post-treatment total psoas area at $L 4$ appears prognostic for tumor recurrence.

\section{Background}

Total body composition (TBC) is an analysis of patient muscle and fat distribution, and represents a global assessment of their state of health. Adverse TBC changes are widespread among cancer patients including those with breast cancer, lung cancer, gastrointestinal malignancies and gynecologic cancer (Awad et al. 2012; Fouladiun et al. 2005; Gil et al. 2006; Harvie et al. 2003; Jacquelin-Ravel and Pichard 2012; Silver et al. 2007; Tan et al. 2009; Smith 2004; Demark-wahnefried et al. 2001). Fluctuations in TBC during cancer treatment are

\footnotetext{
*Correspondence: shrutij@med.umich.edu

${ }^{1}$ Department of Radiation Oncology, University of Michigan, UHB2C447, SPC 5010, 1500 East Medical Center Drive, Ann Arbor, MI 48109-5010, USA Full list of author information is available at the end of the article
}

patient-specific and generally vary according to disease site and treatment modality. Furthermore, TBC changes have been shown to be associated with impairments in physical function (Brown et al. 2005), quality of life, resistance to therapy and worse prognosis in some cancer patient populations (Fearon et al. 2011; Wallengren et al. 2013; Fearon et al. 2006; Couch et al. 2007; Tsai 2012). Given the potential clinical consequences of during and post-treatment $\mathrm{TBC}$ alterations, further understanding these changes and evaluating the potential need for intervention in response to TBC alterations is important.

Head and neck cancer patients, in particular, experience significant treatment and cancer-related weight loss due to a multifactorial metabolic syndrome (Silver et al. 2007). More than $70 \%$ of this weight loss is attributed to loss of muscle mass (Silver et al. 2007), which occurs
Springer Open

C 2016 Wang et al. This article is distributed under the terms of the Creative Commons Attribution 4.0 International License (http://creativecommons.org/licenses/by/4.0/), which permits unrestricted use, distribution, and reproduction in any medium, provided you give appropriate credit to the original author(s) and the source, provide a link to the Creative Commons license, and indicate if changes were made. 
despite adequate nutritional supplementation. This loss of lean muscle mass without a corresponding loss in fat mass, known as sarcopenia, correlates with worse overall prognosis in oropharyngeal cancer patients (Adelstein et al. 2000). Better understanding of TBC in head and neck cancer patients is necessary to design interventions that may improve their quality of life, functional status, and overall prognosis. Moreover, there is need for a convenient and accurate technique to evaluate changes in body composition. Dual-energy X-ray absorptiometry (DEXA) has been the gold standard for determining total body composition; however, its accuracy may be limited in patients undergoing large changes in volume status, such as with head and neck cancer patients undergoing treatment (Jackson et al. 2013). Past studies characterizing changes in TBC have utilized different methods based on fundamentally different techniques, including plain anthropometric measurements (Hyltander et al. 1991; Richards et al. 2012), neutron activation analysis (MacFie and Burkinshaw 1987), bioelectric impedance measurements (Simons et al. 1995), computerized tomography (Smith 2004), and DEXA (Jackson et al. 2013; Maturo et al. 2003; Koch 1998). These methods may be difficult to obtain at the respective institution, and they have yielded inconsistent results due to intrinsic measurement limitations and variations (Fouladiun et al. 2005).

A recently developed novel technique utilizes a high throughput analysis of body composition characteristics (body morphomics analysis) to assess patient frailty. This innovative CT-based approach offers convenience for the patients and limits additional tests as most patients with cancer undergo routine imaging with CT before and after chemoradiation. Furthermore, body morphomics offers significant cost saving implications by reducing additional tests and supports patient-centered individualized cancer care planning.

Body morphomics analysis can rapidly and accurately characterize the changes in regional body composition in cancer patients, and has shown a strong correlation between trunk muscle (psoas) size and mortality in multiple clinical settings, including postoperatively following liver transplant surgery, abdominal aneurysm surgery, and inpatient general surgery (Zarinsefat et al. 2014; Levi et al. 2014; Waits et al. 2014; Englesbe et al. 2013; Sheetz et al. 2013). Core muscle size has similarly shown prognostic value in patients with esophageal cancer (Sheetz et al. 2013), adrenocortical carcinoma (Miller et al. 2012), and melanoma (Sabel et al. 2011). The role of body morphomics in head and neck cancer patients treated with chemoradiotherapy (CRT), who experience significant metabolic disturbances during treatment, remains unexplored to date. Furthermore, to our knowledge no study has looked at the correlation between regional changes in psoas area as determined by $\mathrm{CT}$ with changes in total body composition as determined by DEXA scan, the current gold standard for body composition analysis. This is important to help us understand whether analysis of a single slice $\mathrm{CT}$ is sufficient for predicting changes in total body composition.

In this study of locally advanced head and neck cancer patients undergoing concurrent chemoradiation, we aim to characterize the changes in body morphomics (total psoas area, lean psoas area, psoas muscle density, $\mathrm{HU}$ ) before and after CRT by determining the association between these changes with patient-reported quality of life (QoL) and tumor related outcomes. We hypothesize that decline in psoas area as determined by CT at L4 vertebral body level is associated with worse quality of life and tumor related outcomes. Our second aim was to determine whether changes in psoas area correlate with changes in total body composition as determined by DEXA scan. We hypothesize that psoas area will decline similarly to TBC changes of lean body mass by DEXA scan during CRT.

\section{Methods}

After obtaining University of Michigan Institutional Review Board approval, 93 patients were enrolled in 2 consecutive prospective trials of organ sparing chemotherapy with intensity-modulated radiation therapy (IMRT) for locally advanced oropharyngeal cancer from 2003 to 2011. Eligibility included stages III to IV squamous cell carcinoma of the oropharynx, no prior therapy, Karnofsky performance status $\geq 60$, and primary therapy with CRT. Details of therapy have been previously published (Hunter et al. 2013; Feng et al. 2010). Briefly, all patients required treatment of bilateral neck. All patients received concurrent weekly chemotherapy with carboplatin (area under curve $=1$ ) and paclitaxel $\left(30 \mathrm{mg} / \mathrm{m}^{2}\right)$. None received induction or adjuvant chemotherapy. The IMRT prescription doses were 70 Gy to the primary tumor and involved lymph nodes, 59-63 Gy to high-risk nodal regions and the anatomic compartments around the gross tumor volumes, and 56 Gy to low-risk nodal regions, all delivered in 35 fractions. Priority was given during planning to spare the noninvolved parts of the major salivary glands, the oral cavity, pharyngeal constrictor muscles, larynx, and the esophagus, to minimize late xerostomia and dysphagia. Feeding tubes were inserted if weight loss during therapy approached $10 \%$. All procedures were in accordance with the ethical standards of the committee on human experimentation of the institution or in accord with the Helsinki Declaration of 1975 as revised in 1983. Details of the radiation treatment planning, the dosimetric and functional results in these 
patients have previously been published (Hunter et al. 2013; Feng et al. 2010).

In a separate study, after obtaining University of Michigan Institutional Review Board Approval, 12 patients with locally advanced squamous cell head and neck cancer were enrolled in a prospective study assessing the overall changes in total body composition using DEXA. This cohort is a subset of a larger cohort that is being accrued for a study assessing the impact of exercise training on changes in total body composition for patients receiving concurrent chemoradiation therapy (CCRT) for locally advanced head and neck cancer. Eligibility criteria included patients with American Joint Committee on Cancer stage II-IV squamous cell head and neck cancer receiving CCRT as first-line treatment without surgery. All patients underwent a preliminary nutritional assessment by a dietician before initiation of treatment. Six patients received a percutaneous endoscopoic gastrostomy (PEG) tube before initiation of radiation therapy, and an additional 3 patients received a PEG tube during radiation therapy for enteral nutrition. If a patient experienced $>5$ to $10 \%$ decrease in body mass during the course of treatment, a nutritionist was consulted. All procedures were in accordance with the ethical standards of the committee on human experimentation of the institution or in accord with the Helsinki Declaration of 1975 as revised in 1983. Details of the study have been previously published (Jackson et al. 2013).

Forty-three of ninety-three patients with PET/CT scans obtained pre-treatment and within 3-6 months following completion of treatment, for whom the CT scan included the psoas muscle, were eligible for the present study, and other fifty patients were initially treated at outside institutions and their staging CT scans were not available for analysis. Twelve patients with DEXA-scan based body composition analyses obtained pre-CRT and 2 months post-CRT follow-up were also enrolled (Jackson et al. 2013). Clinical data including age, sex, height, weight, dosimetric data, smoking status, tumor recurrences, mortality, and cancer stage were obtained for the patients.

Body morphomics analysis (BMA) was performed on pre- and post-treatment chest and abdominal computed tomography $(\mathrm{CT})$ in a semi-automated fashion using algorithms programmed in MATLAB v13.0. To determine the cross sectional areas and densities of the left and right psoas muscle at the level of the fourth lumbar vertebra (L4), individual vertebral levels were initially identified on each patient's PET-CT scan in sagittal section. Individual transverse imaging slice at the superior aspect of L4 were selected and the borders of the left and right psoas muscle were outlined using an edge-detecting algorithm, and verified by trained investigators. The areas of the resulting enclosed regions were then computed and summed to yield the total cross-sectional area of the psoas muscle (Englesbe et al. 2013). Average density in Houndsfield Units (HUs) of the psoas muscle in the selected regions was also measured. To adjust for fatty infiltration of the psoas muscle, the average density was used to calculate the total cross-sectional area of the psoas muscles, excluding fatty infiltration (lean psoas muscle) (Englesbe et al. 2012).

Head-and-neck cancer and treatment-related QoL was assessed by 2 validated instruments: the Head and Neck QOL (HNQOL) (Koch 1998) and University of Washington QOL (UWQOL) (Hassan and Weymuller 1993), version 3. Briefly, UWQOL (version 3) is a validated, self-administered, and multi-factorial, questionnaire that provides questions specific to head and neck cancer. Ten questions domains of UWQOL included: pain, appearance, activity, recreation, employment, chewing, speech, taste, saliva, and mucous. Scoring is scaled so that a score of 50 represents the worst possible response, and a score of 10 represents the best possible response. Scoring is scaled in equal increments from ten to fifty to reflect the number of possible responses. Thus the pain domain has 5 possible responses, which are scored as 10, 20, 30, 40, and 50.

The Head and Neck QOL is a validated and selfadministered questionnaire also specific to head and neck cancer. This questionnaire had four domains: eating (6 items), communication (4 items), pain (4 items), and emotion (6 items). Scoring is scaled so that a score of 4 represents the worst possible response, and a score of 0 represents the best possible response. Scoring is scaled in equal increments from 0 to 4 to reflect the five possible responses. For example, within the communication domain, "ability to talk to other people" has 5 possible responses, which are scored as $0,1,2,3$, and 4 . HNQOL and UWQOL questionnaires were administered at 6 time intervals: before initiation of treatment and at 3, 6, 12, 18, and 24 months after treatment.

DEXA measurements of total lean body mass and body morphomics parameters of total psoas area and lean psoas area were the main variables of interest from the respective analysis. Normal distribution of the fore mentioned variables was verified with $\mathrm{Q}-\mathrm{Q}$ plots. BMI categories of normal (18.5-24.9), overweight (25-29.9), and obese $(\geq 30.0)$ were used to stratify for further analysis of weight loss. Changes in weight, BMI, total psoas area, lean psoas area, and lean body mass were characterized with mean, standard deviation, percent change, twosample $t$ test, and $95 \%$ confidence interval. Two-sample $t$ test was employed to compare the percent change of lean body mass with that of total and lean psoas area. Cox proportional hazards model as used to assess the effect 
of total psoas area on tumor recurrence and mortality. The Pearson's correlation coefficient was calculated to evaluate associations between changes in BMA measures from pre-treatment to 3 months post-treatment with changes in UWQOL and HNQOL from pre-treatment to 6 months post-treatment. We chose to analyze the quality of life associations at the fore mentioned time frame because patients were still recovering from CRT side effects at 3 and at 12-24 months, deterioration in quality of life began to return to pre-treatment levels. A significance level of $\alpha=0.05$ was used. All statistical analysis was performed using SPSS (IBM Corp. Released 2013. IBM SPSS Statistics for Windows, Version 22.0. Armonk, NY: IBM Corp.).

\section{Consent}

Written informed consent was obtained from the patients to participate in this study and for publication of this research article and any accompanying images.

\section{Results}

Forty-three of the 93 patients enrolled in prospective phase II chemo-IMRT had pre-treatment and posttreatment follow-up PET/CT scans available for morphomics analysis and thirty-eight of the forty-three had also prospectively completed the UWQOL and HNQOL questionnaires. Twelve other patients had pre- and posttreatment DEXA scans available for total body composition analysis. Characteristics of the two groups are summarized in Table 1. DEXA study patient group and BMA group are similar with respect to age, sex, tumor site, AJCC stage, and IMRT dose. Clinical and demographic similarities between the two groups allow for comparison between total body composition changes and total psoas muscle area changes.

Clinically significant lean muscle mass decline was seen in both BMA and DEXA cohorts, shown in Table 2. Decrease in overall lean body mass by DEXA scan was $10.2 \%$, which is comparable to the degree of decline in total psoas area by body morphomics of $10.9 \%$. Lean psoas area on the other hand seemed to over-estimate the degree of lean body mass loss; it decreased by $14.1 \%$ after CRT. Psoas Houndsfield unit (HU), a measure of muscle density, decreased by $9.2 \%$, which is indicative of increase in fatty infiltration and decline in muscle quality after CRT. Decreases in weight were similar between the DEXA and BMA group (11.7 and $10.33 \%$, respectively). Summary of body morphomics changes are shown in Table 3.

In our BMA cohort, there were nine patients with tumor recurrences and six deaths. In univariate analysis, greater percent decline in total psoas area was correlated with higher risk of tumor recurrence [hazard
Table 1 Clinical characteristics and total body composition changes

\begin{tabular}{lll}
\hline & DEXA study & $\begin{array}{l}\text { Body morphomics } \\
\text { analysis }\end{array}$ \\
\hline Sample size & $\mathrm{N}=12$ & $\mathrm{~N}=43$ \\
Age (years) & $57( \pm 8.1)$ & $57( \pm 7)$ \\
Weight $(\mathrm{kg})$ & $93( \pm 12.6)$ & $92( \pm 17.4)$ \\
BMI & $27.1( \pm 3.0)$ & $29.1( \pm 5)$ \\
LBM (kg) & $59.2( \pm 7.8)$ & - \\
FBM (kg) & $30.6( \pm 7.5)$ & - \\
Total psoas area $\left(\mathrm{mm}^{2}\right)$ & - & $2811( \pm 726)$ \\
Lean psoas area $\left(\mathrm{mm}^{2}\right)$ & - & $1407( \pm 356)$ \\
Male & $12(100.0)$ & $36(95.0)$ \\
Female & $0(0.0)$ & $2(5.0)$ \\
Tumor site & & $20(53.0)$ \\
Base of tongue & $6(50.0)$ & $18(47.0)$ \\
Tonsil & $4(33.3)$ & - \\
Other & $2(16.7)$ & \\
AJCC stage & & $5 \%$ \\
III & $7 \%$ & $95 \%$ \\
IVA & $83 \%$ & - \\
IVB & - & $38(100.0)$ \\
Treatment modality, CCRT & $12(100.0)$ & $70.0( \pm 0.0)$ \\
IMRT dose, Gy & $70.0( \pm 0.0)$ & \\
\hline LBM lean body mass FBM fat & &
\end{tabular}

LBM lean body mass, FBM fat body mass, CCRT concurrent chemo-radiation therapy, Gy gray dosage

ratio $(\mathrm{HR})=1.117 ; 95 \% \mathrm{CI} 1.018,1.225 ; \mathrm{P}=0.019]$ and mortality ( $\mathrm{HR}=1.127 ; 95 \% \mathrm{CI} 1.018,1.225 ; \mathrm{P}=0.019)$, Table 4 . The interpretation is for each $1 \%$ loss of total psoas area, the risk of tumor recurrence and mortality increases by 11.7 and $12.7 \%$ respectively. Given that our patients on average loose $10.9 \%$ of total psoas area, the risk of tumor recurrence and mortality is 127.3 and $138.2 \%$, respectively, higher relative to a patient without any changes in total psoas area.

Higher percent decrease in weight in univariate analyses was correlated with higher risk of mortality $(\mathrm{HR}=1.111 ; 95 \% \mathrm{CI} 1.001,1.233 ; \mathrm{P}=0.047)$ but not tumor recurrence $(\mathrm{P}=0.163)$. As expected, $\mathrm{T}$-stage also correlated with tumor recurrence $(\mathrm{HR}=2.048 ; 95 \% \mathrm{CI}$ 1.024-4.095; $\mathrm{P}=0.043)$ and mortality $(\mathrm{HR}=3.891 ; 95 \%$ CI 1.161-13.046; $\mathrm{P}=0.028$ ) in univariate analyses. The interpretation is for each increase in stage (i.e. from stage III to IV), the risk of tumor recurrence and mortality increases by 104.8 and $289.1 \%$ respectively. Lean psoas area and psoas density (HU) did not correlate with tumor recurrence or mortality in univariate analysis.

In multivariate analysis adjusted for patient age, percent decrease in weight and tumor stage, greater decline in total psoas area was a significant predictor of tumor 
Table 2 DEXA total body composition changes

\begin{tabular}{lccc}
\hline & Net change & Percent change & P value \\
\hline BMI & $1.75( \pm 2.2)$ & 5.96 & $<0.05$ \\
Weight $(\mathrm{kg})$ & $11.2( \pm 6.9)$ & 11.70 & $<0.05$ \\
Lean body mass $(\mathrm{kg})$ & $6.1( \pm 6.8)$ & 10.20 & 0.001 \\
\hline
\end{tabular}

In the DEXA scan cohort, changes in BMI, weight and lean body mass were assessed at pre- and 2 months post-treatment follow-up

recurrence $(\mathrm{HR}=1.148 ; 95 \% \mathrm{CI} 1.005,1.311 ; \mathrm{P}=0.042)$, but not mortality $(\mathrm{P}=0.173)$, Table 5 . While tumor stage was a significant predictor in multivariate analysis of tumor recurrence, when adjusting for total psoas area as well as patient age, and weight, T-stage was not significant in predicting mortality among patients $(\mathrm{HR}=3.054$; 95 \% CI 0.960, 9.717; $\mathrm{P}=0.094$ ). On multivariate analyses adjusted for age, percent decrease in total psoas area, and $\mathrm{T}$-stage, percent decline in weight was not a significant predictor of either tumor recurrence $(P=0.502)$ or mortality ( $\mathrm{P}=0.787)$. Of note, gender was not used as a covariate in multivariate analyses due to small number of females in the cohort $(n=2)$.

AIC scores were calculated to determine the relative contribution of each covariate to model strength. AIC scores in Table 5 reflect model strength when the given covariate is removed from the model; higher AIC indicates greater importance of the removed covariate. Factors with $\mathrm{P}$ value $<0.05$ in the multivariate model are significant predictors of tumor recurrence or mortality. To shed light on the relative contribution of a factor, the AIC was calculated by the removal of that factor from the final model. Higher values of AIC indicates greater importance of the omitted factor. Total psoas
Table 4 Univariate analysis of risk factors and adverse patient outcomes

\begin{tabular}{lll}
\hline Measure & P value & HR (95 \% Cl) \\
\hline (A) Tumor recurrence & & \\
Total psoas area (\% decline) & 0.019 & $1.117(1.018-1.225)$ \\
Weight (\% decline) & 0.163 & $1.064(0.975-1.160)$ \\
Age & 0.43 & $0.961(0.871-1.061)$ \\
T-stage & 0.043 & $2.048(1.024-4.095)$ \\
Lean psoas area (\% decline) & 0.14 & $1.047(0.985-1.114)$ \\
Psoas HU (\% decline) & 0.933 & $0.988(0.957-1.041)$ \\
(B) Mortality & & \\
Total psoas area (\% decline) & 0.043 & $1.127(1.004-1.266)$ \\
Weight (\% decline) & 0.047 & $1.111(1.001-1.233)$ \\
Age & 0.0514 & $1.037(0.929-1.157)$ \\
T-stage & 0.028 & $3.891(1.161-13.046)$ \\
Lean psoas area (\% decline) & 0.147 & $1.060(0.980-1.148)$ \\
Psoas HU (\% decline) & 0.656 & $1.010(0.966-1.057)$ \\
\hline
\end{tabular}

area is a strong predictor, slightly more contributory than T-stage, of tumor recurrence (AIC-Psoas = 44.104; AIC-stage $=43.682$ ).

Decrease in psoas muscle area from pre-treatment to first follow-up was significantly correlated with deterioration in 3 out of 15 UWQOL domains (activity, recreation/entertainment, and swallowing, all $\mathrm{P}<0.05$ ) as well as a single item in HNQOL regarding concerns for cancer related physical problems $(\mathrm{P}<0.05)$ from pre- to 6 months post-treatment, Table 6 . As expected, decline in psoas area are correlated with quality of life domains relating to mobility and maintenance of muscle function (i.e. swallowing). Body weight and BMI are correlated

Table 3 Body morphomics changes

\begin{tabular}{|c|c|c|c|c|}
\hline Characteristics $(\mathrm{N}=43)$ & $\begin{array}{l}\text { Pre-treatment } \\
\text { Mean (SD) }\end{array}$ & $\begin{array}{l}\text { Post-treatment } \\
\text { Mean (SD) }\end{array}$ & Percent change (95 \% Cl) & P value \\
\hline Weight (kg) & $92 \pm 17$ & $82 \pm 15$ & $-10.33(-8.05,-12.61)$ & $<0.001$ \\
\hline Body mass index $(\mathrm{BMI})^{*}$ & $29.1 \pm 5$ & $26.7 \pm 4.2$ & $-9.71(-7.41,-12.01)$ & $<0.001$ \\
\hline Normal $(\mathrm{N}=8)$ & $23.2 \pm 1.14$ & $21.2 \pm 2.89$ & $-8.62(-5.6,-11.64)$ & $<0.05$ \\
\hline Overweight $(N=16)$ & $28.20 \pm 1.41$ & $25.72 \pm 2.03$ & $-8.79(-7.39,-10.19)$ & $<0.001$ \\
\hline Obese $(\mathrm{N}=19)$ & $34.71 \pm 3.18$ & $29.86 \pm 3.03$ & $-13.97(-11.7,-16.24)$ & $<0.001$ \\
\hline Bone Marrow Density (L4) & $159 \pm 42.1$ & $159 \pm 41.3$ & 0 & - \\
\hline Total psoas area $\left(\mathrm{mm}^{2}\right)$ & $2811 \pm 726$ & $2492 \pm 626$ & $-10.88(-8.70,-13.06)$ & $<0.001$ \\
\hline Left psoas area $\left(\mathrm{mm}^{2}\right)$ & $1407 \pm 356$ & $1244 \pm 311$ & $-10.75(-7.73,-13.77)$ & $<0.001$ \\
\hline Right psoas area $\left(\mathrm{mm}^{2}\right)$ & $1404 \pm 385$ & $1248 \pm 328$ & $-10.74(-8.45,-13.03)$ & $<0.001$ \\
\hline Psoas density (HU) & $52.0 \pm 7.1$ & $47.2 \pm 7.9$ & $-9.16(-4.42,-13.9)$ & $<0.005$ \\
\hline Lean psoas $\left(\mathrm{HU} \times \mathrm{mm}^{2}\right)$ & $1130 \pm 300$ & $973 \pm 270$ & $-14.09(-11.07,-17.01)$ & $<0.001$ \\
\hline
\end{tabular}

Changes in body morphomics measures were assessed from pre-treatment to 3 months post-up

* BMI categories of normal (18.5-24.9), overweight (25-29.9) and obese ( $\geq 30)$ were used to stratify the cohort 
Table 5 Mulitivariate analysis of risk factors and adverse patient outcomes

\begin{tabular}{|c|c|c|c|c|c|c|}
\hline \multirow[t]{2}{*}{ Factor (\% decrease) } & \multicolumn{3}{|l|}{ Tumor Recurrence } & \multicolumn{3}{|l|}{ Mortality } \\
\hline & Hazard ratio $(95 \% \mathrm{Cl})$ & $\mathbf{P}$ & AIC & Hazard ratio $(95 \% \mathrm{Cl})$ & $\mathbf{P}$ & AIC \\
\hline Total psoas area & $1.148(1.005-1.311)$ & 0.042 & 44.104 & $1.111(0.955-1.292)$ & 0.173 & 40.657 \\
\hline Weight & $0.966(0.875-1.068)$ & 0.502 & 41.496 & $1.016(0.908-1.136)$ & 0.787 & 40.341 \\
\hline Age & $0.909(0.805-1.026)$ & 0.909 & 41.723 & $1.037(0.891-1.207)$ & 0.643 & 40.308 \\
\hline T-stage & 2.305 (1.059-5.018) & 0.035 & 43.682 & $2.878(0.836-9.914)$ & 0.094 & 41.387 \\
\hline
\end{tabular}

Table 6 Summary of body morphomics and quality of life correlations

\begin{tabular}{|c|c|c|c|c|c|c|}
\hline & \multicolumn{2}{|l|}{ Body weight } & \multicolumn{2}{|c|}{ Body mass index } & \multicolumn{2}{|c|}{ Psoas muscle } \\
\hline & Pearson's r & $P$ value & Pearson's r & P value & Pearson's r & $P$ value \\
\hline \multicolumn{7}{|l|}{ UWQOL } \\
\hline Activity & -0.219 & 0.213 & -0.189 & 0.284 & $\underline{-0.399}$ & $\underline{0.019}$ \\
\hline Chewing & -0.324 & 0.062 & -0.289 & 0.097 & -0.284 & 0.104 \\
\hline Disfigurement & -0.033 & 0.853 & 0.077 & 0.665 & 0.197 & 0.264 \\
\hline Employment & -0.056 & 0.753 & -0.0004 & 0.998 & 0.033 & 0.853 \\
\hline General pain & -0.404 & $\underline{0.018}$ & -0.374 & $\underline{0.029}$ & -0.226 & 0.198 \\
\hline Mouth pain & -0.21 & 0.233 & -0.197 & 0.264 & -0.137 & 0.44 \\
\hline Mucus amount & -0.132 & 0.456 & -0.119 & 0.503 & -0.239 & 0.173 \\
\hline Mucus consistency & 0.091 & 0.609 & -0.08 & 0.653 & 0.014 & 0.937 \\
\hline Recreation/entertainment & -0.423 & $\underline{0.013}$ & -0.378 & $\underline{0.028}$ & -0.438 & $\underline{0.0096}$ \\
\hline Saliva amount & -0.031 & 0.862 & 0.0001 & 0.999 & -0.048 & 0.787 \\
\hline Saliva consistency & 0.159 & 0.369 & 0.217 & 0.218 & 0.157 & 0.375 \\
\hline Speech & -0.398 & $\underline{0.0197}$ & -0.37 & $\underline{0.031}$ & -0.252 & 0.151 \\
\hline Swallowing & -0.240 & 0.172 & -0.226 & 0.199 & -0.401 & $\underline{0.019}$ \\
\hline Taste & -0.338 & $\underline{0.05}$ & -0.306 & 0.078 & -0.066 & 0.711 \\
\hline Throat Pain & -0.194 & 0.272 & -0.2 & 0.256 & -0.152 & 0.291 \\
\hline \multicolumn{7}{|l|}{ UM HNQOL } \\
\hline \multicolumn{7}{|c|}{ Emotion domain: concerns regarding... } \\
\hline Cancer related physical problems & -0.143 & 0.419 & -0.129 & 0.467 & -0.453 & 0.007 \\
\hline
\end{tabular}

with 4 out of 14 UWQOL domains (general pain, recreation/entertainment, speech, and taste, all $\mathrm{P}<0.05$ ) and 3 out of 14 UWQOL domains (general pain, recreation/entertainment, and speech, all $\mathrm{P}<0.05$ ) respectively from pre- to 6 months post-treatment. No significant correlations were found between body weight and BMI with HNQOL. Summary of the correlations of changes in body morphomics with quality of life are shown in Table 6.

It is notable that tumor stage did not yield significant correlations with either HNQOL or UWQOL from preto 6 months post-treatment. Furthermore, common toxicity criteria (Trotti et al. 2000) also did not yield significant correlations with HNQOL or UWQOL from pre to 6 months post-treatment.

\section{Discussion}

This is the first study to analyze the changes in body composition through a novel analytic morphomics technique utilizing single slice PET/CT at L4 in oropharyngeal cancer patients undergoing chemoradiation. We investigated the potential for using total psoas area by body morphomics to measure TBC changes. The study found similar declines in total lean body mass by DEXA, the current gold standard for TBC measurements (Jackson et al. 2013), and total psoas area by body morphomics, indicating that perhaps single slice CT total psoas area at L4 may adequately capture TBC lean body mass changes. Furthermore, body morphomics is performed on routine staging CT scans, offering patients convenience and limits the need for additional testing. 
This study found significant declines in total psoas area, lean psoas area, and psoas HU from pre-treatment to 3 months post-treatment follow-up. Psoas muscle area decline is clinically significant because it correlates with deterioration in patient reported physical activity (3 out of 15 UWQOL domains) and concerns regarding cancer related physical problems (1 out of 4 HNQOL domains) from pre- to 6 months post-treatment on univariate analysis. Furthermore, decline in total psoas area independently predicts tumor recurrence in univariate and multivariate analysis adjusted for age, tumor stage, and decline in weight. In multivariate analysis, psoas muscle area loss also appears to be a greater contributor to tumor recurrence than T-stage, similar to results reported by Sheetz et al. (2013) who found lean psoas area to be a greater contributor than stage.

We found that lean psoas area and psoas density (HU) did not correlate with tumor recurrence or mortality in univariate analysis. This finding is in contrast to results reported by Sheetz et al. who conducted a similar study with esophageal cancer patients undergoing esophagectomy. They found lean psoas area to be predictive of overall survival and disease free survival on univariate analysis and multivariate analysis (Sheetz et al. 2013). This discrepancy shows that the sensitivities of different body morphomics measures (total psoas area vs. lean psoas area) in different clinical settings are not fully understood and require further research.

This study also found significant changes in body weight from pre-CRT baseline to post-CRT, a degree of loss that is comparable to findings from similar studies (Jackson et al. 2013). From our study, the effects of weight loss appear to hold less clinical significance than decline in total psoas area. Decline in body weight did not predict tumor recurrence in univariate or multivariate analysis. On univariate analysis, weight loss is associated with greater risk of mortality; however, this relationship is not significant on multivariate analysis adjusted for age, percent decline in total psoas area, and T-stage. In the multivariate models, psoas area also appears to be a stronger predictor for tumor recurrence than weight loss. Weight loss correlated with deterioration in general quality of life (i.e. general pain, recreation and entertainment, taste, and speech) domains from pre- to 6 months post-treatment on univariate analysis. This is in contrast with quality of life deterioration associated with total psoas area, which are specific to frailty and mobility.

There is a need for measurements of BMI to better quantify the interpersonal variations in the significance of weight loss (Fearon et al. 2006) and few studies have examined the changes in BMI in cancer patients in general. We examined the changes in BMI in oropharyngeal cancer patients and found that on average BMI decreased by $9.7 \%$. By stratifying patients into BMI categories of obese, overweight, and normal, we found distinct patterns of weight losses specific to each group. Decrease in BMI correlated with declines in 3 out of 15 UWQOL domains from pre- to 6 months post-treatment on univariate analysis. There is significant overlap in the associated QOL domains between BMI and weight loss. This indicates that future studies may only need to focus on one or the other when characterizing quality of life outcomes.

Our finding that total psoas area predicted mortality in oropharyngeal cancer patients on univariate analysis is not surprising given total psoas area decline is associated with increased mortality in multiple clinical settings (Zarinsefat et al. 2014; Levi et al. 2014; Waits et al. 2014; Englesbe et al. 2013; Sheetz et al. 2013; Miller et al. 2012; Sabel et al. 2011). It is surprising that decline in psoas area independently predicted tumor recurrence adjusted for age, T-stage, and weight loss. This is the first study to our knowledge to delineate this relationship. It is possible that patients who are more likely to experience tumor recurrence may have declines in body composition that portends the recurrence and can be readily detected by body morphomics; thus, in that subset of patients, body composition changes is reflectively of ongoing metabolic and inflammatory derangements of the patients.

Despite these findings, there are several limitations in this study. The study cohort represents a subset of previously published patients (Feng et al. 2010); our cohort size was limited by inclusion criteria of having pre- and post-treatment PET/CT as well as prospective completion of UWQOL and HNQOL questionnaires. Furthermore, our sample size $(n=38)$ limits the possibility of multivariate analysis for quality of life correlations. Future studies with larger patient cohorts are required to better delineate the relationship between decline in total psoas and deterioration in quality of life. Such studies may allow clinicians to prospectively identify patients who should undergo exercise and nutritional interventions to improve quality of life into survivorship (Jackson et al. 2013).

In conclusion, to better aid in the understanding of changes in detailed body composition, body morphomics analysis is an accurate and convenient method for patients to characterize body composition changes in cancer patients using the patient's staging CT (or PET/ CT) scans. Notably, body morphomics appears to be a more sensitive measure of adverse outcomes (i.e. tumor recurrences) than tumor stage and weight loss. Body morphomics is also associated with clinically significant changes in QOL up to 6 months post-CRT.

Given the accessibility of data in already existing staging and follow-up CT scans of head and neck cancer 
patients and ability to accurately provide body composition data using body morphomics analysis, this methodology may prove to be a vital instrument in the longitudinal evaluation of cancer patients. It could help clinicians identify patients who are at increased risk for cachexia as well as monitor the effects of behavioral interventions (diet and exercise) in cancer patients. More research in body morphomics in cancer patients is necessary to better understand its broad clinical application.

\section{Authors' contributions}

CW participated in the design of the study, performed the statistical analysis, and helped to draft the manuscript. JV helped to draft the manuscript and advised on interpretation on results. MV helped with data acquisition. PR helped with data acquisition. JS helped with data acquisition. SW conceived the underlying analytical technique applied in this paper and helped to draft the manuscript. AE helped to draft the manuscript. SJ conceived the study, participated in the design of the study, and helped to draft the manuscript. All authors read and approved the final manuscript.

\section{Author details}

1 Department of Radiation Oncology, University of Michigan, UHB2C447, SPC 5010, 1500 East Medical Center Drive, Ann Arbor, MI 48109-5010, USA.

${ }^{2}$ Morphomic Analysis Group, University of Michigan, Ann Arbor, MI, USA.

${ }^{3}$ Department of Radiation Oncology, Veterans Affairs Ann Arbor Health Care System, Ann Arbor, MI, USA.

\section{Acknowledgements}

The authors thank Center for Statistical Consultation and Research of the University of Michigan for statistical support.

Portions of this study have been presented in abstract form at the annual Multidisciplinary Head and Cancer Symposium, Phoenix, AZ, on February 20, 2014.

\section{Competing interests}

The authors declare that they have no competing interests.

Received: 20 January 2016 Accepted: 30 March 2016

Published online: 11 April 2016

\section{References}

Adelstein DJ, Lavertu P, Saxton JP et al (2000) Mature results of a phase III randomized trial comparing concurrent chemoradiotherapy with radiation therapy alone in patients with stage III and IV squamous cell carcinoma of the head and neck. Cancer 88:876-883

Awad S, Tan BH, Cui H et al (2012) Marked changes in body composition following neoadjuvant chemotherapy for oesophagogastric cancer. Clin Nutr 31:74-77

Brown DJF, McMillan DC, Milroy R (2005) The correlation between fatigue, physical function, the systemic inflammatory response, and psychological distress in patients with advanced lung cancer. Cancer 103:377-382

Couch M, Lai V, Cannon T et al (2007) Cancer cachexia syndrome in head and neck cancer patients: part I diagnosis, impact on quality of life and survival, and treatment. Head Neck 19:2381-2389

Demark-wahnefried W, Peterson BL, Winer EP et al (2001) Changes in weight, body composition, and factors cancer patients receiving adjuvant chemotherapy. J Clin Oncol 19:2381-2389

Englesbe MJ, Lee JS, He K et al (2012) Analytic morphomics, core muscle size, and surgical outcomes. Ann Surg 256:255-261

Englesbe MJ, Terjimaian MN, Lee JS et al (2013) Morphometric age and surgical risk. J Am Coll Surg 216:976-985

Fearon KC, Voss AC, Hustead DS (2006) Definition of cancer cachexia: effect of weight loss, reduced food intake, and systemic inflammation on functional status and prognosis. Am J Clin Nutr 83:1345-1350

Fearon K, Strasser F, Anker SD et al (2011) Definition and classification of cancer cachexia: an international consensus. Lancet Oncol 12:489-495
Feng FY, Kim HM, Lyden TH et al (2010) Intensity-modulated chemoradiotherapy aiming to reduce dysphagia in patients with oropharyngeal cancer: clinical and functional results. J Clin Oncol 28:2732-2738

Fouladiun M, Korner U, Bosaeus I, Daneryd P, Hyltander A, Lundholm KG (2005) Body composition and time course changes in regional distribution of fat and lean tissue in unselected cancer patients on palliative care - correlations with food intake, metabolism, exercise capacity, and hormones. Cancer 103:2189-2198

Gil KM, Frasure HE, Hopkins MP, Jenison EL, von Gruenigen VE (2006) Body weight and composition changes in ovarian cancer patients during adjuvant chemotherapy. Gynecol Oncol 103:247-252

Harvie MN, Campbell IT, Thatcher N, Baildam A (2003) Changes in body composition in men and women with advanced nonsmall cell lung cancer (NSCLC) undergoing chemotherapy. J Hum Nutr Diet 16:323-326

Hassan SJ, Weymuller EA (1993) Assessment of quality of life in head and neck cancer patients. Head Neck 15:485-496

Hunter KU, Schipper M, Feng FY et al (2013) Toxicities affecting quality of life after chemo-IMRT of oropharyngeal cancer: prospective study of patientreported, observer-rated, and objective outcomes. Int J Radiat Oncol Biol Phys 85:935-940

Hyltander A, Drott C, Unsgaard B et al (1991) The effect on body composition and exercise performance of home parenteral nutrition when given as adjunct to chemotherapy of testicular carcinoma. Eur J Clin Invest 21:413-420

Jackson W, Alexander N, Schipper M, Fig L, Feng F, Jolly S (2013) Characterization of changes in total body composition for patients with head and neck cancer undergoing chemoradiotherapy using dual-energy $X$-ray absorptiometry. Head Neck 36:1356-1362

Jacquelin-Ravel N, Pichard C (2012) Clinical nutrition, body composition and oncology: a critical literature review of the synergies. Crit Rev Oncol Hematol 84:37-46

Koch J (1998) The role of body composition measurements in wasting syndromes. Semin Oncol 25:9-12

Levi B, Zhang P, Lisiecki J et al (2014) Use of morphometric assessment of body composition to quantify risk of surgical-site infection in patients undergoing component separation ventral hernia repair. Plast Reconstr Surg 133:559e-564e

MacFie J, Burkinshaw L (1987) Body composition in malignant disease. Metabolism 36:290-294

Maturo G, Vespasiani G, Mohamed EL, Maiolo C et al (2003) Evaluating body composition of Italian prostate cancer patients without metastases. Acta Diebetol 40:s168-s170

Miller BS, Ignatoski M, Daignault S et al (2012) Worsening central sarcopenia and increasing intra-abdominal fat correlate with decreased survival in patients with adrenocortical carcinoma. World J Surg 36:1509-1516

Richards CH, Roxburgh CSD, MacMillan MT et al (2012) The relationships between body composition and the systemic inflammatory response in patients with primary operable colorectal cancer. PLoS ONE 7:e41883

Sabel MS, Lee J, Cai S et al (2011) Sarcopenia as a prognostic factor among patients with stage III melanoma. Ann Surg Oncol 18:3579-3585

Sheetz KH, Zhao L, Holcombe SA et al (2013) Decreased core muscle size is associated with worse patient survival following esophagectomy for cancer. Dis Esophagus 7:716-722

Silver HJ, Dietrich MS, Murphy BA (2007) Changes in body mass, energy balance, physical function, and inflammatory state in patients with locally advanced head and neck cancer treated with concurrent chemoradiation after low-dose induction chemotherapy. Head Neck 10:893-900

Simons JP, Schols AM, Westerterp KR, Ten Velde GP, Wouters EF (1995) The use of bioelectrical body water in patients impedance with cancer analysis to predict total body water in patients with cancer cachexia. Am J Clin Nutr 61:741-745

Smith MR (2004) Changes in fat and lean body mass during androgen-deprivation therapy for prostate cancer. Urology 63:742-745

Tan BHL, Birdsell LA, Martin L, Baraco VE, Fearon KCH (2009) Sarcopenia in an overweight or obese patient is an adverse prognostic factor in pancreatic cancer. Clin Cancer Res 15:6973-6979

Trotti A, Byhardt R, Stetz J et al (2000) Common toxicity criteria: version 2.0. an improved reference for grading the acute effects of cancer treatment: impact on radiotherapy. Int J Radiat Oncol Biol Phys 47:13-47

Tsai S (2012) Importance of lean body mass in the oncologic patient. Nutr Clin Pract 27:593-598 
Waits SA, Kim EK, Terjimanian MN et al (2014) Morphometric age and mortality after liver transplant. JAMA Surg 149:335-340

Wallengren O, Lundholm K, Bosaeus I (2013) Diagnostic criteria of cancer cachexia: relation to quality of life, exercise capacity and survival in unselected palliative care patients. Support Care Cancer 21:1569-1577
Zarinsefat A, Terjimanian MN, Sheetz KH et al (2014) Perioperative changes in trunk musculature and postoperative outcomes. J Surg Res 191:106-112

\section{Submit your manuscript to a SpringerOpen ${ }^{\odot}$} journal and benefit from:

- Convenient online submission

- Rigorous peer review

- Immediate publication on acceptance

- Open access: articles freely available online

- High visibility within the field

- Retaining the copyright to your article

Submit your next manuscript at $\gg$ springeropen.com 